\title{
Solution to Transport Chain Sustainability: Increasing E-Commerce User Awareness
}

\author{
Akriti Kartik, Gauri Jere, Kritman Singh, Reuben Sam, Yash Kumar Singhal
}

\begin{abstract}
With an increase in global sourcing and online shopping, the negative effects of an unsustainable supply chain are becoming evident. We have designed an algorithm for an online plug-in to help combat this problem. The algorithm computes and processes the carbon emissions during the delivery of each product. Using this data, a 'Sustainability Rating' out of five, would be generated to help consumers make better informed choices.

To understand our idea's potential effects, we collected primary data about consumer patterns and preferences through a survey. Our analysis of this data showed that consumers would make greener choices if they were aware of the environmental impact of their purchases.

As customers would opt for products with higher sustainability ratings, our solution can, in the long term, shift the consumer base towards more localized deliveries and significantly reduce the carbon footprint of online shopping.

The research and ideation in our paper can be used to further develop our project, and possibly implement it in the future.

Index Terms - Transport Chain, online shopping.
\end{abstract}

\section{INTRODUCTION}

Over the years, online shopping has become more and more convenient and thus extremely popular. But although one day deliveries and free returns are very helpful, there is a big cost that comes along with the E-Commerce industry: a huge carbon footprint.

Avid customers of online shopping platforms ourselves, we decided to work on a way to resolve this problem. After reading through some statistics, we were able to recognise that the gas emissions from transport vehicles make up a large part of the carbon footprint. If we could make this part of the process more sustainable it would help lead to an overall improvement.

Our initial idea was to work on the technological aspect of transport and help design vehicles or engines that run on renewable energy sources or have modifications that help reduce gas emissions. As students, this wasn't very feasible due to our lack of resources and knowledge in the area. Thus, we decided to switch gears and instead target the deliveries themselves. We understood that if the customer base would make greener choices, it would act as a catalyst to help increase the sustainability of the entire transport chain. For example, if one user is choosing between two brands of cookies and decides to order the one that is stored at a warehouse closer to the user's location, the transport distance reduces and thus so do the gas emissions during the delivery. If this same scenario were to occur at a larger scale, over time it would help promote more localised deliveries and make a big dent in the transport supply chain's carbon footprint.

To allow the customers to choose more sustainable products, they need to have carbon footprint data readily available to them. So, we designed a system that would store, organise, and deliver this data to the customer in a user friendly and easily comprehensible form: a sustainability rating out of 5 .

I. The Code and the Algorithm

\section{A. The API Used in The Code}

Our algorithm utilises an API (Application Programming Interface) called the 'Distance Matrix API' from Google. It functions similarly to Google Maps; for a matrix of origin and destination points, the API will automatically compute the distance to be travelled, the time that will be taken, all based on the recommended route it determines. This API is crucial to our code, as it calculates the distances based on the pre-existing roads and routes of the area. If we were to use a regular distance calculator, the output would actually be the displacement (the shortest distance possible) rather than the actual path that would be travelled, due to factors such as roads and highways.

Hence, our algorithm would have to include this API. The pricing of the API is 5.00 USD per 1000 iterations. A rough estimation would determine the use of the algorithm to be expensive; Amazon sells millions of products - each would count to be at least 1 iteration of the API. At a bare minimum, assuming Amazon only sells a million products, the pricing would still be around 5000.00 USD.

\section{B. Database Concept and Maintenance}

We decided that the best way for the program to function efficiently was to organize all the required information in a database. This database would consist of a Unique Product ID for each deliverable product along with its respective warehouse location.

This way the program would be able to effectively search for the product closest to the consumer, and then proceed on its journey. However, this system would require the database to constantly be updated as goods would move through different warehouses. 
We realize this would be a tedious process to do manually and the obvious solution would be to figure out a method of automation. Let us call one complete run of the algorithm, 1 cycle. 1 cycle of the code would consist of all the calculations required to determine the optimum route and is only completed when the product has been moved to the new address. The code identifies which product is being purchased and removes the current warehouse address and inputs the new address at the time of arrival at the next warehouse. Hence, in transit, a product would not be able to be traced to a specific location. This prevents redundancy of data, as otherwise a product would be stored at two locations at once. Since the API estimates the time taken, the database could be refreshed with the new address after the time period has elapsed with some buffer time.

\section{Determination of Modes OfTransport Used:}

Most online retailers use TMS (transport Management Software), which calculates the most effective delivery routes in real-time. We had an idea to use old TMS databases to train a machine learning software to accurately assume routes and calculate sustainability using that data. Unfortunately, accessing those databases proved to be difficult. So, we instead decided to stick by our initial assumption, that products will move between warehouses and then to the final location. Although this would not be as accurate, our algorithm would still provide a good approximation of the sustainability of the product's transportation.

Our code was designed to work in India, for regular deliveries (not accounting for one-day deliveries or urgent deliveries). To accurately determine the different modes of transport used, the algorithm would utilise the distance and time taken (calculated by the API) to determine the average speed of the transport used. This speed could be used to determine the mode of transport that the package travelled via - if the speed is anywhere from 0 to $60 \mathrm{kmph}$ (inclusive), the mode of transport can be approximated to be a truck, and if the speed is anywhere above $60 \mathrm{kmph}$, the transport will be assumed to be via train. As this code only considers deliveries across India, these two modes of transport are usually favoured. For the code to be developed for other regions, the possibility of travelling over water should be considered. In that situation, the only two methods of transport would be ship and plane. The constants for these would be around $200 \mathrm{kmph}$. If the speed is between 0 and $200 \mathrm{kmph}$, the mode of transport could be considered a ship. If it exceeds $200 \mathrm{kmph}$, it would be considered to be a plane.

\section{D.Determination ofThe Sustainability Rating}

The database would now have the mode of transport as well as the distance travelled. Hence, by using standard constants for carbon emissions per vehicle, the average carbon emissions per delivery could be calculated. We designed a rating system (1-5 bands from Good to Bad), which computes all the different emissions ever calculated, and compares the current value against them. Judging by this, a rating between 1-5 will be determined. The specific bands are created to allow consumers to interpret this data easily. Each time the code is iterated, and the approximate calculation is made, it will be added to the database as well. Hence, with each iteration, the algorithm will become more accurate with time. The initial data would need to be calculated before the launch of the plugin itself, but once this is done, the database and ratings should run smoothly.

\section{The Research and Analysis}

\section{A. Aim}

To test whether our solution could actually work, we had to observe consumer patterns with and without the aforementioned "Sustainability Rating".

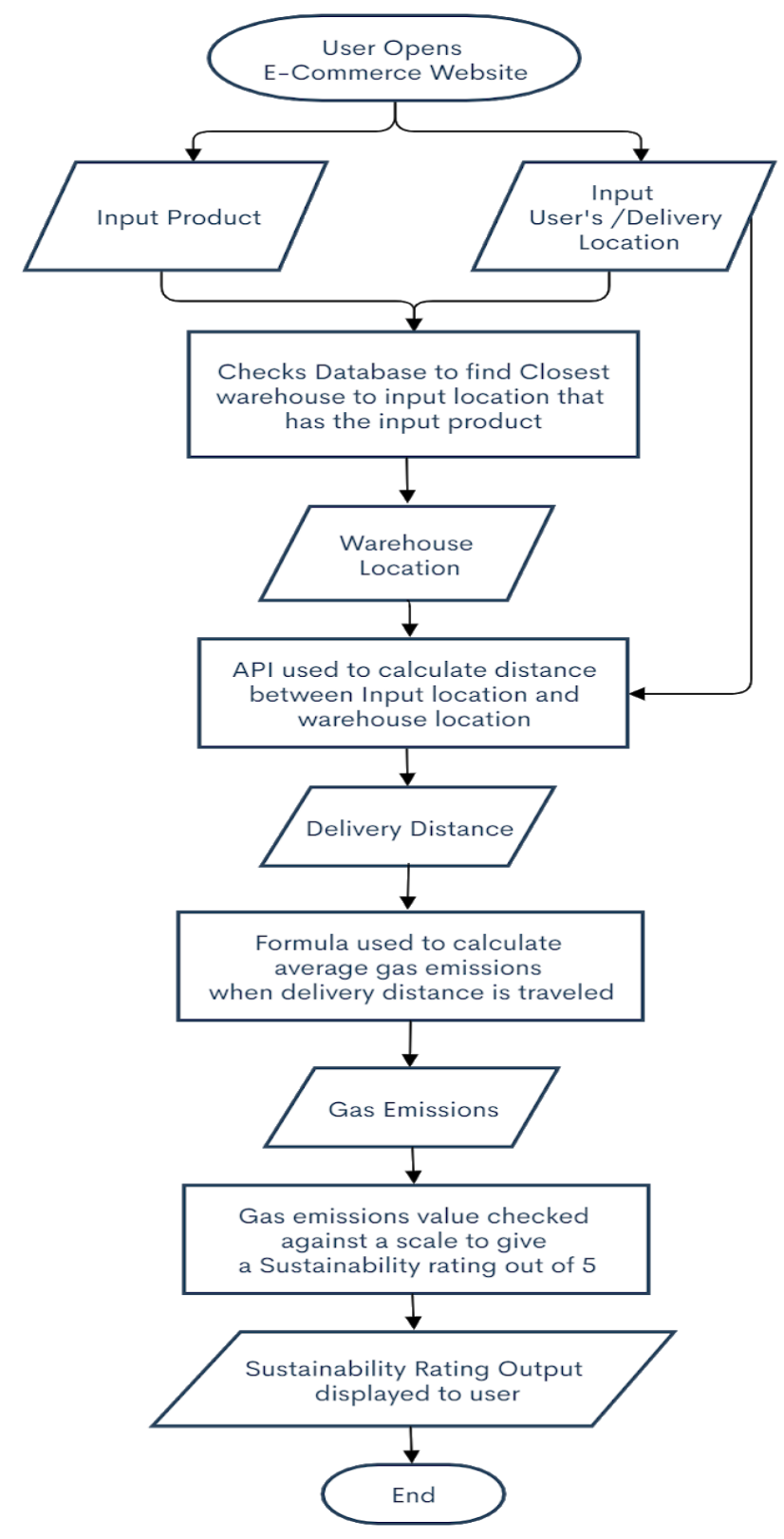

\section{B. Hypothesis:}

If a customer has access to data about the environmental impact of multiple products, they will opt for the more sustainable option. 


\section{Research Method:}

Questionnaire: we decided to simulate an online shopping interface using a Google Form.

\section{Sampling}

We shared the google form with our peers, teachers, family members and amongst the communities we are part of. Thus, our participant base was via Convenience Sampling. We were able to collect 78 responses from people spanning a large range of age groups and an almost equal number of male and female participants (and 3 others who didn't want to share their gender). And the results from this survey are represented in fig 4.1 in the next column.

\section{E. Experiment Design}

This form comprised 17 questions:

The first two questions were to collect demographic data such as age, group, and gender.

We had recognised four main factors that a consumer would
The final question was a blatant inquiry about which factor was the most significant to the participant. This allowed us to test their explicit and implicit shopping habits.

\section{F. Strength}

Wehad a diverse demographic of participants thus increasing generalizability. The form included many control questions where all three choices had the same or similar price, brand, and sustainability rating to understand how much influence personal preference would have on responses and to take note of individual bias amongst the participants. We also had some questions with completely random choices so as to try to hide our aim from the participants in order to avoid inaccurate responses.

To ensure that there wasn't a bias towards choosing more sustainable options out of fear of societal pressures, we allowed the participants to remain anonymous, which gave them the opportunity to vote honestly.

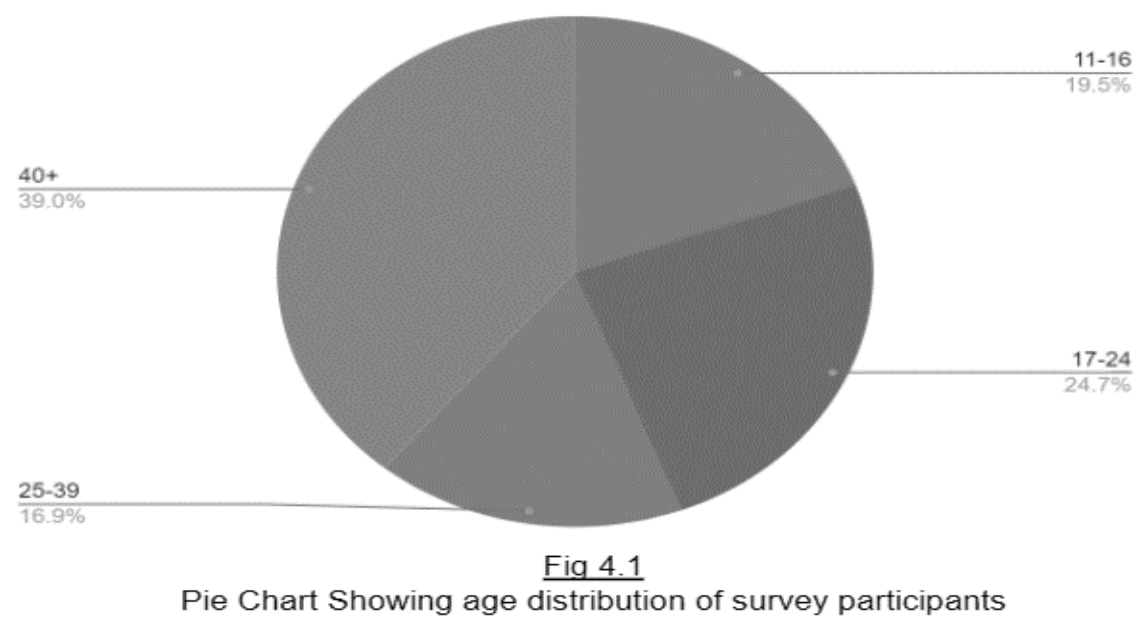

consider when weighing product options: price, personal

\section{G.Limitations}

preference, brand, and the sustainability rating given to it. We chose biscuits as our product, as there are many variations of this item spanning a large range of costs and brands, allowing us to test with flexibility. The options in each question were made such that each question tested one or two of the factors while keeping the rest constant.

The form was then made to have 14 decision-based questions. In each of these fourteen, there were three different biscuit/cookie options. Each option included the type of biscuit, its brand, its cost (in rupees), and a sustainability rating. These questions would help us understand which factor had the highest weightage on average among the participants.

Our participant range did not span multiple socio-economic strata as it was targeted towards the mid-high socio-economic categories. This may lead to unreliable conclusions as the entire E-commerce consumer base was not considered.

Using a questionnaire also had its own limitations. Since the answers are not filled when under observation, the participant may lose interest mid-way and answer randomly, leading to discrepancies in the results. Participants may also be able to figure out the true aim of our experiment and alter their answers. Ecological validity may reduce as our Google form did not look like an actual online shopping interface that the surveyed would be familiar with. 


\section{H.Results}

Through analysis of the responses, we were able to understand the purchasing patterns and preferences of an average consumer base. The results showed two general trends:

(1) over $48 \%$ of the time, a consumer would choose the more sustainable product, giving less weight to the price or their preference

(2) Many a time, the consumer would choose a product of relatively intermediate or a 'Medium' price and/or sustainability.

\section{Analysis of Data}

Through the survey, results obtained suggested that a significant number of users would consider the implications of the carbon footprint left behind due to their product delivery and chose the more sustainable option out of the product choices given to them.

The survey had a series of questions where either one, two or three of the factors (price, brand and sustainability rating) were varied. Using this, it was determined that consumers pay the most heed to brand, with sustainability following behind it.

To prove that this product would be useful, certain questions in the survey were structured to see if the sustainability rating had any influence on a consumer's choice. Through these questions, it was inferred that $44.8-51.3 \%$ went for the most sustainable option, with over $22 \%$ of them saying that they paid attention to the sustainability rating provided.

Start on a smaller scale. Take Karnataka for instance. It has a population of 67 million people. If even $30 \%$ of them chose a more sustainable option, that would be 13.4 million people making a difference. Now increase the scale - in India itself, 330 million people shop online. If $20 \%$ of them used a sustainability rating, that would be 66 million people. The scale of impact would be massive and would go a long way towards reducing emissions during the chain of transport.

\section{CONCLUSION}

Recently, the e-commerce industry has started to grow at an astonishing rate. The Covid-19 pandemic has only hastened this change, going as far as to leave online shopping as the only option for some. This, however, leads to a major problem: the supply chain for this system was not designed for such a large demand, and becomes unsustainable for the amount and type of use it gets today.

Analysis of the data from the questionnaire we crafted, aimed at the type of person likely to make up the bulk of online purchases, shows that the carbon footprint of the purchase is the second largest factor in influencing consumer decisions. If this algorithm could actually be developed and utilised, it could modify the chain of transportation for the better.

Unfortunately, as high school students, developing such a plug-in is beyond our financial capabilities, and so we hope that anyone with more access to resources who wishes to drive a change in the e-commerce industry can take this idea, and make it what it has the potential to be.

\section{REFERENCES}

[1] Google Cloud Platform, Google, console.cloud.google.com/apis/library/distance-matrix-backend.googl eapis.com?filter=category:maps\&id=82aa0d98-49bb-4855-9da9-efde 390a3834\&project=distance-matrix-api-273213\&authuser $=3 \&$ folder \&organizationId.

[2] Carbon Footprint Ltd using RADsite CMS (www.radsite.co.uk/). "Carbon Offset Options." Offset Quotation, www.carbonfootprint.com/offset.aspx $? 0=4400000$.

[3] "Country Facts." IndexMundi, www.indexmundi.com/.

[4] "Ecommerce 101: The Impact, History \&amp; Future of Electronic Commerce." The BigCommerce Blog, 30 July 2020, www.bigcommerce.com/blog/ecommerce/\#the-future-of-ecommerce.

[5] Farmbrough, Heather. "Why Internet Shopping Isn't Always Better For the Environment." Forbes, Forbes Magazine, 14 Oct. 2019, www.forbes.com/sites/heatherfarmbrough/2019/10/14/why-internet-sh opping-isnt-always-better-for-the-environment/\#2e0879d15c18.

[6] “Green Rating Systems.” HDR, 17 May 2017, www.hdrinc.com/services/sustainability-resiliency/green-rating-syste ms.

[7] “The History Of Ecommerce: How Did It All Begin?" Miva Blog, 15 Apr. 2020,

www.miva.com/blog/the-history-of-ecommerce-how-did-it-all-begin/.

[8] "International Green Construction Code (IgCC)." ICC, 13 June 2019, www.iccsafe.org/products-and-services/i-codes/2018-i-codes/igcc/.

[9] "Life Cycle Databases." Life Cycle Databases | Greenhouse Gas Protocol, ghgprotocol.org/life-cycle-databases.

[10] Naterattner. "This Map Shows How Amazon's Warehouses Are Rapidly Expanding across the Country." CNBC, CNBC, 19 Jan. 2020, www.cnbc.com/2020/01/19/map-of-amazon-warehouses.html.

[11] Rohrer, Jürg, et al. "CO2 Emissions for Shipping of Goods." Time for Change, 12 Oct. 2019, timeforchange.org/co2-emissions-for-shipping-of-goods/

[12] Sarah DeWeerdt for Conservation magazine, part of the Guardian Environment Network. "How Green Is Online Shopping?" The Guardian, Guardian News and Media, 17 Feb. 2016, www.theguardian.com/environment/2016/feb/17/how-green-is-onlineshopping.

[13] Wicker, Alden. "No Online Shopping Company Can Figure out How to Quit This One Plastic Bag.” Vox, Vox, 31 Jan. 2019, www.vox.com/the-goods/2019/1/31/18203972/polybags-plastic-online -shopping-meal-kits-patagonia.

[14] Wulfraat, Marc. "Amazon Supply Chain and FulfillmentCenter Network." Amazon Distribution Network Strategy | MWPVL International, mwpvl.com/html/amazon_com.html. 


\begin{abstract}
Akriti Kartik, I am Akriti Kartik, a 17-year-old student looking to pursue science. I have always been fascinated by the way things work, be it a machine or a living organism. Apart from that, I enjoy reading, especially the fantasy and mystery genres. I love immersing myself in other worlds, that being the main reason for my binge watching of marvel and action movies. I also enjoy water activities, especially swimming and Scuba Diving, and I have dived in Australia, and two places in India. Another hobby of mine is art - the pages of my notebook and paint stains on my floor can attest to that. I look forward to making meaningful change and contributions towards saving the environment in any way I can.
\end{abstract}

Gauri Jere,I love trying to figure out how the world works, and with that comes a lot of science. Studying chemistry, physics, and biology allow me to explore these interests, and I plan to pursue Chemical engineering at an undergraduate level. I'm also an avid reader and writer. I write short stories and poetry when I'm not busy spending all my time on YouTube.

Kritman Singh, I love all things STEM!I'm all for those abstract mathematical concepts, and love putting science to the test by applying it in the real world! I plan to get into a more research-oriented career, using what I've learned across all sciences. I'm a former member of Inventure Academy, now part of Manthan. I'm also a member of the New York Academy of Science's Junior Academy and have many more papers in the works!

Reuben Sam John. I am a high school student in my final year at Inventure academy. I am an astrophile; I enjoy learning about the universe and the laws governing it. I plan to pursue astrophysics for my undergraduate studies and plan to move on to research. Aside from space, my other interests include, playing football. I've been playing for many years and have represented my school for numerous tournaments. and to flex my creative muscles, I also enjoy playing guitar, playing live any opportunity I get, or simply just to relax.

Yash Kumar Singhal, I'm currently a high school student at Inventure Academy, pursuing my A levels. I am passionate about all things science and spend my time experimenting both in my school's labs and in my kitchen. I plan to pursue a degree in Science and takeLife Sciences / Biology as my undergraduate major to get into a research career. I'm a budding science communicator and can often be found researching for my blog, "Ridiculously Random Rambles" or planning episodes for my podcast, "Crazy Classroom Conversations".https://ridiculouslyrandomrambles.wordpress.com/pages/ 